Bull. Soc. math. France

129 (2), 2001, p. 237-257

\title{
FONCTEURS POLYNOMIAUX ET FONCTEURS DE MACKEY NON LINÉAIRES
}

\author{
par Hans-Joachim Baues, Winfried Dreckmann, \\ Vincent Franjou \& Teimuraz Pirashyili
}

RÉSumÉ. - On décrit les foncteurs polynomiaux, des groupes abéliens libres vers les groupes abéliens, comme des diagrammes de groupes abéliens dont on explicite les relations.

Abstract (Polynomial functors and nonlinear Mackey functors)

Polynomial functors from free abelian groups to abelian groups are described explicitely in the form of diagrams of abelian groups, that are maps between the crosseffects of the polynomial functor which satisfy a list of relations. The key is to use an appropriate notion of Mackey functor from the category of finite sets and surjections.

Soient $\mathcal{A} b$ la catégorie des groupes abéliens, et $F(\mathbb{Z})$ sa sous-catégorie pleine dont les objets sont les groupes abéliens libres de type fini. On sait que la catégorie des foncteurs additifs de $F(\mathbb{Z})$ vers $\mathcal{A} b$ est équivalente à la catégorie $\mathcal{A} b$.

Texte reçu le 25 avril 2000, accepté le 25 septembre 2000

Hans-JoAChim BaUes, Max-Planck-Institut für Mathematik, Vivatsgasse 7, 53111 Bonn

(Germany) - E-mail : baues@mpim-bonn.mpg.de

Winfried Dreckmann, Schlatwieske 26, 48282 Emsdetten, Germany

E-mail : WD@lidingo.mail.telia.com

Vincent Franjou, UMR 6629 UN/CNRS, Université de Nantes, Département de mathématiques, 2 rue de la Houssinière, BP 92208, 44322 Nantes CEDEX 3 (France)

E-mail : franjou@math.univ-nantes.fr

Teimuraz Pirashvili, A.M. Razmadze Mathematical Institute, Aleksidze str. 1, Tbilisi 380093 (Republic of Georgia) • E-mail : pira@mpim-bonn.mpg.de

Classification mathématique par sujets (2000). — 18D, 20C12, 20C30, 20G05, 55U, 55P15. T.P. : bourse INTAS-93-2618-Ext, membre du réseau TMR "K-theory and algebraic groups" ERB FMRX CT-97-010. 
En fait, un foncteur additif $T: F(\mathbb{Z}) \rightarrow \mathcal{A} b$ est entièrement déterminé par sa valeur sur $\mathbb{Z}$, et le foncteur $T$ est isomorphe au produit tensoriel par $T(\mathbb{Z})$ :

$$
T \cong(-) \otimes T(\mathbb{Z}) \text {. }
$$

Soit $\mathcal{F}(\mathbb{Z})$ la catégorie de tous les foncteurs de $F(\mathbb{Z})$ vers $\mathcal{A} b$ qui s'annulent en 0 . Pour un entier positif $n$, on désigne par $\mathcal{P}_{n}(\mathbb{Z})$ la sous-catégorie pleine de $\mathcal{F}(\mathbb{Z})$ dont les objets sont les foncteurs polynomiaux de degré inférieur ou égal à $n$ au sens d'Eilenberg et Mac Lane [6] (voir $\S 1)$. Quand $n$ vaut 1 , il s'agit des foncteurs additifs, et l'on vient d'expliquer que la catégorie $\mathcal{P}_{1}(\mathbb{Z})$ est équivalente à la catégorie $\mathcal{A} b$. Une description de la catégorie $\mathcal{P}_{2}(\mathbb{Z})$ a été obtenue par H. Baues [2]. Elle est équivalente à la catégorie des diagrammes de groupes abéliens

$$
M_{e} \stackrel{H}{\longrightarrow} M_{e e} \stackrel{P}{\longrightarrow} M_{e}
$$

vérifiant les relations $P H P=2 P$ et $H P H=2 H$. La correspondance s'effectue à partir d'un foncteur quadratique $T$ en posant

$$
M_{e}=T(\mathbb{Z}) \quad \text { et } \quad M_{e e}=T(\mathbb{Z} \mid \mathbb{Z}) .
$$

Le but principal de cet article est de décrire les catégories $\mathcal{P}_{n}(\mathbb{Z})$ en termes simples, et de les présenter comme catégories de diagrammes de groupes abéliens.

On peut consulter [2] pour une interprétation homotopique des foncteurs quadratiques. Signalons aussi que les groupes d'extensions dans les catégories de foncteurs sont accessibles au calcul, et admettent des interprétations très riches au-delà même de l'article [9]. Pour une généralisation de nos résultats et des applications aux algèbres de Schur, on pourra consulter [7].

Nous terminons cette introduction par la description de nos résultats. Nous commencerons par généraliser la notion de degré d'un foncteur polynomial ( $\$ 1$ ). Pour les besoins de l'article, il nous suffit de considérer les foncteurs, vers la catégorie des groupes abéliens, dont la catégorie source possède un objet nul et admet des coproduits finis. C'est bien sûr le cas de la catégorie $F(\mathbb{Z})$, mais c'est aussi le cas de la catégorie des monoïdes commutatifs libres de type fini, catégorie que l'on désignera par $F(\mathbb{N})$. Ces deux catégories sont reliées par le foncteur de complétion en groupe de Grothendieck; par précomposition, on obtient un foncteur :

$$
\mathcal{F}(\mathbb{Z}) \longrightarrow \mathcal{F}(\mathbb{N})
$$

(la notation au but se comprend d'elle-même) qui n'est pas une équivalence de catégorie, mais qui le devient une fois restreint aux sous-catégories des foncteurs polynomiaux $(\S 8)$. La description de $\mathcal{P}_{n}(\mathbb{Z})$ s'obtient d'un résultat plus général concernant la catégorie $\mathcal{F}(\mathbb{N})$. Cette dernière est en effet équivalente à la catégorie des foncteurs de Mackey [5] des ensembles finis vers les groupes abéliens. On cherche alors à appliquer le théorème principal de [12] (voir $\S 2$ ). Il s'agit de simplifier un foncteur défini sur les ensembles finis en un foncteur

TOME $129-2001-\mathrm{N}^{\mathrm{O}} 2$ 
défini sur les seules surjections. L'énoncé du résultat réclame que l'on dispose de la notion de foncteur de Mackey dans le cas des seules surjections entre ensembles finis, notion que l'on définit ci-dessous (et au $\S 5$ ).

Dans cet article, un foncteur de Janus $M$ d'une catégorie $\mathcal{C}$ vers une catégorie $\mathcal{A}$ est la donnée d'un foncteur covariant $M_{*}$ et d'un foncteur contravariant $M^{*}$ de $\mathcal{C}$ vers $\mathcal{A}$ qui coïncident sur les objets (pour Dress [5], c'est un bifoncteur, terme que nous réservons à un foncteur de deux variables; l'expression "foncteur de Janus" est due à B. Richter).

Soit $\Omega$ la catégorie des ensembles finis non-vides et des surjections.

DÉFInition 0.1. - Un foncteur de Janus $M$, de $\Omega$ vers $\mathcal{A} b$, est un foncteur de Mackey si il vérifie la condition suivante : pour tout diagramme dans $\Omega$,

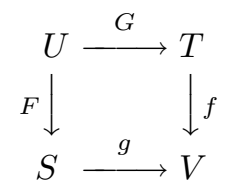

qui est un carré cartésien d'ensembles, on a, dans le groupe $\operatorname{Hom}(M(S), M(T))$,

$$
M^{*}(f) M_{*}(g)=\sum M_{*}\left(g^{\prime}\right) M^{*}\left(f^{\prime}\right)
$$

où la somme s'effectue sur les sous-ensembles $X$ de $U$ tels que les restrictions $f^{\prime}$ et $g^{\prime}$ de $F$ et $G$ à $X$ restent des surjections.

On peut maintenant énoncer nos principaux résultats.

THÉORÈme 0.2. - La catégorie $\mathcal{F}(\mathbb{N})$ de tous les foncteurs, de la catégorie $F(\mathbb{N})$ des monö̈des commutatifs libres de type fini vers $\mathcal{A} b$, qui envoient 0 sur 0 , est équivalente à la catégorie des foncteurs de Mackey, de la catégorie $\Omega$ des surjections entre ensembles finis vers $\mathcal{A} b$.

THÉORÈME 0.3. - Soit $n$ un entier positif. La catégorie $\mathcal{P}_{n}(\mathbb{Z})$ des foncteurs de degré inférieur ou égal à $n$, de la catégorie $F(\mathbb{Z})$ des groupes abéliens libres de type fini vers abéliens $\mathcal{A} b$, est équivalente à la catégorie des foncteurs de Mackey, de $\Omega$ vers $\mathcal{A} b$, qui s'annulent sur les ensembles ayant plus de $n$ éléments.

Le dernier paragraphe explicite une présentation des catégories $\mathcal{P}_{n}(\mathbb{Z})$.

Remerciements. - Les premier et quatrième auteurs remercient l'Université de Nantes pour son hospitalité.

BULletin DE LA SOCiÉtÉ MATHÉMATIQUE DE FRANCE 


\section{Foncteurs polynomiaux}

Soit $\mathcal{A}$ une catégorie qui possède un objet nul, noté 0 , et qui admet des coproduits finis. On note

$$
i_{1}: X \longrightarrow X \vee Y, \quad i_{2}: Y \longrightarrow X \vee Y
$$

les inclusions de deux objets dans leur coproduit, et

$$
r_{1}=\left(1_{X}, 0\right): X \vee Y \longrightarrow X, \quad r_{2}=\left(0,1_{Y}\right): X \vee Y \longrightarrow Y
$$

les rétractions.

Pour tout foncteur $F$ de $\mathcal{A}$ vers $\mathcal{A} b$ tel que $F(0)=0$, sa déviation, ou seconde déviation, est le foncteur de deux variables défini sur un couple $(X, Y)$ par :

$$
F(X \mid Y):=\operatorname{Ker}\left(F(X \vee Y) \stackrel{\left(r_{1}, r_{2}\right)}{\longrightarrow} F(X) \times F(Y)\right) .
$$

Comme le morphisme de projection $\left(r_{1 *}, r_{2 *}\right): F(X \vee Y) \rightarrow F(X) \times F(Y)$ admet une section donnée par : $(x, y) \mapsto i_{1 *}(x)+i_{2 *}(y)$, la suite exacte courte :

$$
0 \rightarrow F(X \mid Y) \longrightarrow F(X \vee Y) \longrightarrow F(X) \times F(Y) \rightarrow 0
$$

est scindée et on a un isomorphisme naturel en $(X, Y)$ :

$$
F(X \vee Y) \cong F(X) \oplus F(Y) \oplus F(X \mid Y)
$$

Pour définir la troisième déviation, on peut alors considérer $F(X \mid Y)$ comme un foncteur en $X$, et en prendre la déviation. On définit par itération les déviations d'ordre supérieur. On vérifie que la $n$-ième déviation d'un foncteur $F$ est :

$$
F\left(X_{1}|\cdots| X_{n}\right)=\operatorname{Ker}\left(F\left(X_{1} \vee \cdots \vee X_{n}\right) \rightarrow \bigoplus_{i=1}^{n} F\left(X_{1} \vee \cdots \vee \hat{X}_{i} \cdots \vee X_{n}\right)\right)
$$

Un foncteur polynomial est un foncteur dont l'une des déviations est nulle.

On dit qu'un foncteur est de degré $n$ si sa $(n+1)$-ième déviation est nulle, mais pas sa $n$-ième.

\section{Un théorème de Dold-Kan pour les $\Gamma$-groupes abéliens}

On énonce dans ce paragraphe le résultat démontré dans [12]. Ici, et dans tout l'article, on considère deux catégories dont les objets sont des ensembles finis. La catégorie $\Gamma$ est la catégorie des ensembles finis pointés. Pour un entier positif $n$, on notera $[n]$ l'ensemble $\{0,1, \ldots, n\}$ basé en 0 . On convient que la somme [1] $\vee \cdots \vee[1]$ de $n$ copies de [1] égale $[n]$. La catégorie $\Omega$ est plus simplement la catégorie des ensembles finis non vides et des surjections. On notera $\underline{n}$ l'ensemble $\{1, \ldots, n\}$.

Si $T$ est un $\Gamma$-groupe abélien, c'est-à-dire un foncteur

$$
T: \Gamma \longrightarrow \mathcal{A} b
$$

TOME $129-2001-\mathrm{N}^{\mathrm{O}} 2$ 
tel que $T([0])=0$, on définit un foncteur

$$
\operatorname{cr}(T): \Omega \longrightarrow \mathcal{A} b
$$

de la manière suivante. La valeur $\operatorname{de} \operatorname{cr}(T)$ sur l'objet $\underline{n}$ est celle de la $n$-ième déviation de $T$ en $([1], \ldots,[1])$ :

$$
\operatorname{cr}(T)(\underline{n}):=T([1]|\cdots|[1]) .
$$

C'est un sous-groupe de $T([1] \vee \cdots \vee[1])=T([n])$. Pour une surjection $f$ : $\underline{n} \rightarrow \underline{m}$, on définit $\operatorname{cr}(T)(f)$ de la manière suivante. Soit $f_{0}:[n] \rightarrow[m]$ l'unique extension de $f$. On voit que l'image de $\operatorname{cr}(T)(\underline{n})$ dans $T([m])$ par le morphisme $\left(f_{0}\right)_{*}: T([n]) \rightarrow T([m])$ est contenue dans $\operatorname{cr}(T)(\underline{m})$. On prend pour $\operatorname{cr}(T)(f)$ le morphisme induit par restrictions de $\left(f_{0}\right)_{*}$.

THÉORÈme 2.1. - La correspondance $T \mapsto \operatorname{cr}(T)$ définit une équivalence de catégorie :

$$
\operatorname{cr}: \mathcal{A} b^{\Gamma} \longrightarrow \mathcal{A} b^{\Omega} \text {. }
$$

La formule

$$
T([m])=\bigoplus_{\mu \subset \underline{m}} \operatorname{cr}(T)(\underline{|\mu|})
$$

où $|\mu|$ désigne le cardinal d'un sous-ensemble $\mu$ de $\underline{m}$, nous dit comment décrire un quasi-inverse pour le foncteur cr. Si $T$ désigne cette fois un foncteur de $\Omega$ vers $\mathcal{A} b$, on définit pour tout entier $m$ positif non nul

$$
i_{!}(T)([m])=\bigoplus_{\mu \subset \underline{m}} T \underline{(|\mu|)},
$$

et pour une application pointée $f:[m] \rightarrow[n]$, on définit

$$
i_{!} T(f): i_{!} T([m]) \longrightarrow i_{!} T([n])
$$

comme le morphisme dont la restriction à un facteur $T(\underline{|\mu|)}$ est nulle si 0 est dans l'image $f(\mu)$ et est le morphisme

$$
f_{*}: T\left(\underline { | \mu | ) } \longrightarrow T \left(\underline{|f(\mu)|)} \subset i_{!} T([n])\right.\right.
$$

induit par $f$ si 0 n'est pas dans $f(\mu)$.

On constate que les foncteurs de $\Gamma$ vers $\mathcal{A} b$ de degré inférieur ou égal à $n$ correspondent, sous cette équivalence, exactement aux foncteurs de $\Omega$ vers $\mathcal{A} b$ qui s'annulent sur les ensembles ayant plus de $n$ éléments.

Concluons ce paragraphe en indiquant qu'une version contravariante de ce résultat est valide : la correspondance $T \mapsto \operatorname{cr}(T)$ définit aussi une équivalence de catégorie

$$
\mathrm{cr}: \mathcal{A} b^{\Gamma \mathrm{op}} \longrightarrow \mathcal{A} b^{\Omega \mathrm{op}} \text {. }
$$

BULletin DE LA SOCiÉtÉ MATHÉMATIQUE DE FRANCE 
On obtient un quasi inverse $i_{*}$ en posant encore, pour un foncteur contravariant $T: \Omega \rightarrow \mathcal{A} b$, et pour tout entier $m$ positif non nul :

$$
i_{*}(T)([m])=\bigoplus_{\mu \subset \underline{m}} T(\underline{|\mu|}),
$$

et en définissant, pour une application pointée $f:[m] \rightarrow[n]$ :

$$
i_{*} T(f): i_{*} T([n]) \rightarrow i_{*} T([m])
$$

comme le morphisme dont la restriction à un facteur $T(|\nu|)$ est nulle vers les facteurs $T(\underline{|\mu|)}$ tels que $f(\mu) \neq \nu$, et est le morphisme

$$
f_{*}: T(\underline{|\nu|}) \longrightarrow T(\underline{|\mu|})
$$

induit par $f$ vers les facteurs $T(\underline{|\mu|})$ tels que $f(\mu)=\nu$.

\section{Monoïdes commutatifs}

Ce paragraphe rappelle les notations nécessaires au maniement des monoïdes. On note Mon la catégorie des monoïdes commutatifs. On sait qu'elle possède une structure de catégorie monoïdale fermée pour le produit suivant. Pour deux monoïdes commutatifs $A$ et $B$, le monoïde $A \otimes B$ est le monoïde commutatif engendré par les couples $a \otimes b$ de $A \times B$ soumis aux relations engendrées par

$$
a \otimes\left(b_{1}+b_{2}\right)=a \otimes b_{1}+a \otimes b_{2}, \text { et }\left(a_{1}+a_{2}\right) \otimes b=a_{1} \otimes b+a_{2} \otimes b .
$$

Les monoïdes $\mathbb{N} \otimes A$ et $A \otimes \mathbb{N}$ sont isomorphes à $A$. L'ensemble des morphismes de $A$ vers $B$ possède une structure naturelle de monoïde commutatif, et on a la formule d'adjonction

$$
\operatorname{Hom}(A, \operatorname{Hom}(B, C)) \cong \operatorname{Hom}(A \otimes B, C) .
$$

Le foncteur d'inclusion de la catégorie $\mathcal{A} b$ dans $\mathcal{M}$ on admet un adjoint à gauche, connu sous les noms de complétion en groupe ou construction de Grothendieck, que l'on note gr. En fait, on a

$$
\operatorname{gr}(A) \cong \mathbb{Z} \otimes A \text { et } \operatorname{gr}(A \otimes B)=\operatorname{gr}(A) \otimes \operatorname{gr}(B) .
$$

Ayant ainsi équipé $\mathcal{M}$ on d'une structure de catégorie monoïdale fermée, on peut considérer les monoïdes vis-à-vis de cette structure, que l'on appelle semianneaux. Il s'agit donc d'anneaux, à ceci près que la structure sous-jacente n'est pas celle d'un groupe abélien, mais celle d'un monoïde commutatif. D'ailleurs, pour un semi-anneau $R$, sa complétion $\operatorname{gr}(R)$ est un anneau. Les $R$-objets à gauche ou à droite sont respectivement appelés semi-modules à gauche ou à droite. Les semi-modules à gauche forment une catégorie, qui est semi-additive au sens rappelé au paragraphe suivant.

TOME $129-2001-\mathrm{N}^{\mathrm{O}} 2$ 


\section{Catégories semi-additives}

DÉFInItion 4.1. - Une catégorie semi-additive est une catégorie $\mathcal{A}$ avec un objet nul, enrichie sur la catégorie des monoïdes commutatifs, et qui possède des biproduits finis. Autrement dit, pour tous $X, Y$ de $\mathcal{A}$, l'ensemble des morphismes $\operatorname{Hom}(X, Y)$ est muni d'une structure de monoïde commutatif telle que la composition est bi-additive, et, à tout couple d'objets $X_{1}, X_{2}$ de $\mathcal{A}$, sont associés un objet $X_{1} \oplus X_{2}$ et deux couples de morphismes

$$
i_{k}: X_{k} \rightarrow X_{1} \oplus X_{2} \quad \text { et } \quad r_{k}: X_{1} \oplus X_{2} \rightarrow X_{k}, \quad k=1,2,
$$

tels que

$$
i_{1} r_{1}+i_{2} r_{2}=1_{X_{1} \oplus X_{2}}, \quad r_{1} i_{1}=1_{X_{1}}, \quad r_{2} i_{2}=1_{X_{2}}, \quad r_{1} i_{2}=0 \quad \text { et } \quad r_{2} i_{1}=0 .
$$

Il est clair que $X \oplus Y$ est alors à la fois produit et coproduit de $X$ et de $Y$. En fait (voir [1, chap. 1, prop. 3.1]) :

Proposition 4.2. - Soit $\mathcal{A}$ une catégorie avec un objet nul, qui possède produits et coproduits finis. La catégorie $\mathcal{A}$ est semi-additive si et seulement si le morphisme

$$
\left(1_{X}, 0\right) \sqcup\left(0,1_{Y}\right): X \sqcup Y \longrightarrow X \times Y
$$

est un isomorphisme.

Un objet $X$ d'une catégorie semi-additive est un générateur si tout objet est isomorphe au coproduit d'un nombre fini de copies de $X$. Soit par exemple un semi-anneau $R$. La catégorie des $R$-semi-modules à gauche est semi-additive. La plus grande sous-catégorie additive dont l'objet $R$ est un générateur est la catégorie $F(R)$ des $R$-semi-modules libres de type fini. Cet exemple est universel : si $X$ est un générateur d'une catégorie semi-additive $\mathcal{A}$, celle-ci est équivalente à la catégorie $F(R)$ pour le semi-anneau $\operatorname{End}_{\mathcal{A}}(X)^{\mathrm{op}}$.

\section{Foncteurs de Mackey}

Ce paragraphe a pour but d'adapter $[5, \S 2]$ et [10] à la catégorie $\Omega$, qui ne possède pas de produit fibré.

DÉFinition 5.1. - Soit $\mathcal{C}$ une catégorie et soient $f: B \rightarrow A, g: C \rightarrow A$ une paire de morphismes dans $\mathcal{C}$ de même but. Un carré au-dessus de $(f, g)$ est un diagramme commutatif de $\mathcal{C}$ :

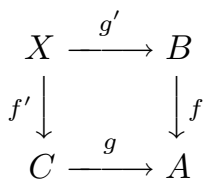

BULLETIN DE LA SOCIÉTÉ MATHÉMATIQUE DE FRANCE 
Une famille cartésienne est un ensemble $C(f, g)$ de carrés au-dessus de $(f, g)$ vérifiant la propriété suivante : pour tout carré $X$ au-dessus de $(f, g)$, il existe un unique morphisme de $X$ vers un carré de $C(f, g)$.

DÉfinition 5.2. - On dit qu'une catégorie $\mathcal{C}$ possède des produits fibrés faibles si toute paire de morphismes de même but possède une famille cartésienne finie non vide.

DÉfinition 5.3. - Soit $\mathcal{C}$ une catégorie avec produits fibrés faibles et soit $\mathcal{A}$ une catégorie semi-additive. Un foncteur de Mackey de $\mathcal{C}$ vers $\mathcal{A}$ est un foncteur de Janus $M$ qui vérifie les deux propriétés suivantes :

(i) Pour tout isomorphisme $f$, la composée $M_{*}(f) \circ M^{*}(f)$ est l'identité

(ii) Pour toute paire de morphismes de même but $f: B \rightarrow A, g: C \rightarrow A$, il existe une famille cartésienne $C(f, g)$ telle que

$$
M^{*}(f) M_{*}(g)=\sum_{X \in C(f, g)} M_{*}\left(g^{\prime}\right) M^{*}\left(f^{\prime}\right)
$$

dans le monoïde $\mathcal{A}(M(C), M(B))$, où l'on note

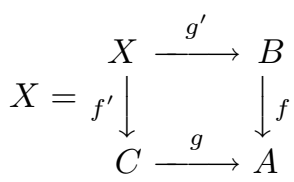

La condition (i) assure que la condition (ii) ne dépend pas de la famille cartésienne choisie.

La catégorie $\Omega$ possède des produits fibrés faibles : pour un carré cartésien d'ensembles finis,

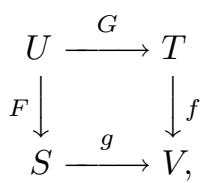

$U=S \times_{V} T$, où $f$ et $g$ sont des surjections, la famille $C(f, g)$ est indexée par les sous-ensembles $X$ de $U$ tels que les restrictions $f^{\prime}$ et $g^{\prime}$ de $F$ et $G$ à $X$ restent des surjections. Dans le cas où la source est la catégorie $\Omega$, on retrouve donc la définition 0.1 donnée dans l'introduction.

Remarque 5.4. - Dans le cas où la catégorie $\mathcal{C}$ admet des produits fibrés et des coproduits, Dress [5] utilise l'expression "pre-Mackey functor", et réserve "Mackey functor" aux foncteurs de Mackey linéaires, au sens où

$$
\left(M^{*}\left(i_{1}\right), M^{*}\left(i_{2}\right)\right): M(X \sqcup Y) \longrightarrow M(X) \oplus M(Y),
$$

TOME $129-2001-\mathrm{N}^{\mathrm{O}} 2$ 
$i_{1}: X \rightarrow X \sqcup Y$ et $i_{2}: Y \rightarrow X \sqcup Y$ désignant les inclusions de deux objets dans leur coproduit, est un isomorphisme. Nous verrons que nous avons précisément besoin de foncteurs de Mackey, non plus linéaires, mais polynomiaux.

On généralise maintenant une construction de Bénabou [3, 7.2 appliqué à 2.6]. Soit $\mathcal{C}$ une catégorie avec produits fibrés faibles. Pour deux objets $S$ et $T$, on appelle flèche de $S$ vers $T$ une classe d'équivalence de diagrammes

$$
S \stackrel{f}{\longleftarrow} V \stackrel{g}{\longrightarrow} T,
$$

pour la relation qui rend équivalents deux diagrammes

$$
S \stackrel{f}{\longleftarrow} V \stackrel{g}{\longrightarrow} T \text { et } S \stackrel{f^{\prime}}{\longleftarrow} V^{\prime} \stackrel{g^{\prime}}{\longrightarrow} T
$$

si il existe un diagramme commutatif

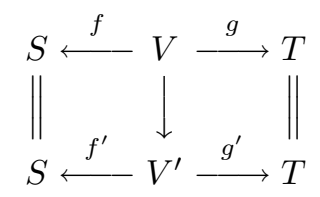

tel que le morphisme $V \rightarrow V^{\prime}$ soit un isomorphisme de $\mathcal{C}$.

On construit une catégorie $\mathbb{N} \mathcal{Q C}$ de la manière suivante. Ses objets sont ceux de $\mathcal{C}$. Les morphismes de $S$ vers $T$ dans $\mathbb{N} \mathcal{Q C}$ sont les sommes de flèches dans le monoïde libre qu'elles engendrent. On peut en effet définir la composée de deux flèches

$$
S \stackrel{h}{\longleftarrow} V \stackrel{k}{\longrightarrow} T \quad \text { et } \quad R \stackrel{f}{\longleftarrow} U \stackrel{g}{\longrightarrow} S
$$

par la somme

$$
\sum_{X \in C(g, h)} R \stackrel{f h^{\prime}}{\longleftarrow} X \stackrel{k g^{\prime}}{\longrightarrow} T .
$$

On vérifie que, pour un objet $S$, le diagramme $S \stackrel{1_{S}}{\longleftarrow} S \stackrel{1_{S}}{\longleftrightarrow} S$ est un morphisme identité. L'associativité de cette composition découle de la formule suivante. Si $S \stackrel{\ell}{\longleftarrow} W \stackrel{m}{\longrightarrow} T, R \stackrel{h}{\longleftarrow} V \stackrel{k}{\longrightarrow} S$ et $Q \stackrel{f}{\longleftarrow} U \stackrel{g}{\longrightarrow} R$ sont trois flèches composables, leur composée est égale à

$$
\sum_{Z \in C\left(g^{\prime}, \ell^{\prime}\right)}\left(\sum_{C(g, h) \times C(k, \ell)} Q \stackrel{f h^{\prime} \ell^{\prime \prime}}{\longleftarrow} \underset{m k^{\prime} g^{\prime \prime}}{\longrightarrow} T\right) .
$$

Les morphismes de $\mathcal{C}$ donnent lieu naturellement à des flèches : pour un morphisme $f: S \rightarrow T$ dans $\mathcal{C}$, on définit

$$
i_{*}(f): S \stackrel{1_{S}}{\longleftarrow} S \stackrel{f}{\longrightarrow} T \text { et } i^{*}(f): T \stackrel{f}{\longleftarrow} S \stackrel{1_{S}}{\longrightarrow} S .
$$

On constate :

Lemme 5.5. - Toute flèche $S \stackrel{f}{\longleftarrow} V \stackrel{g}{\longrightarrow} T$ est la composée $i_{*}(g) i^{*}(f)$.

BULLETIN DE LA SOCiÉTÉ MATHÉMATIQUe DE FRANCE 
On vérifie alors, comme dans le cas usuel, les résultats suivants. Bien sûr, $\mathcal{C}$ y désigne une catégorie avec produits fibrés faibles.

Proposition 5.6. - La correspondance $i_{*}$ (respectivement $i^{*}$ ) définit un foncteur covariant (resp. contravariant) de $\mathcal{C}$ vers $\mathbb{N} \mathcal{Q C}$.

Proposition 5.7. - La correspondance qui, à une flèche $S \stackrel{f}{\longleftarrow} V \stackrel{g}{\longrightarrow} T$, asssocie la flèche $T \stackrel{g}{\longleftarrow} V \stackrel{f}{\longrightarrow} S$, s'étend en un foncteur additif contravariant $D$ de $\mathbb{N} \mathcal{Q C}$ vers $\mathbb{N} \mathcal{Q C}$. Ce foncteur est involutif, si bien que la catégorie $\mathbb{N} \mathcal{Q C}$ est auto-duale.

ThÉORÈme 5.8. - Soit $\mathcal{C}$ une catégorie avec produits fibrés faibles et soit $\mathcal{A}$ une catégorie semi-additive. La catégorie des foncteurs de Mackey de $\mathcal{C}$ vers $\mathcal{A}$ est équivalente à la catégorie des foncteurs additifs de $\mathbb{N} \mathcal{Q C}$ vers $\mathcal{A}$.

En guise de démonstration, contentons-nous d'indiquer la correspondance. Si $M: \mathbb{N} \mathcal{Q C} \rightarrow \mathcal{A}$ est un foncteur additif, on définit un foncteur de Janus en posant

$$
M_{*}(f):=M\left(i_{*}(f)\right), \quad M^{*}(f):=M\left(i^{*}(f)\right) .
$$

Inversement, si $M$ est un foncteur de Janus de $\mathcal{C}$ vers $\mathcal{A}$, on définit

$$
M(S \stackrel{f}{\longleftarrow} V \stackrel{g}{\longrightarrow} T):=M_{*}(g) M^{*}(f) .
$$

Notons que dans le cas d'une catégorie avec produits fibrés, la composée de deux flèches est encore une flèche, si bien que l'on obtient déjà une catégorie $\mathcal{Q C}$ avec les seules flèches pour morphismes. Ceci permet d'éviter les décorations de l'énoncé précédent :

THÉORÈme 5.9 (voir [10]). — Soit $\mathcal{C}$ une catégorie avec produits fibrés et soit $\mathcal{A}$ une catégorie semi-additive. La catégorie des foncteurs de Mackey de $\mathcal{C}$ vers $\mathcal{A}$ est équivalente à la catégorie des foncteurs de $\mathcal{Q C}$ vers $\mathcal{A}$.

\section{6. À propos de la catégorie $\mathcal{Q E} n s$}

Ce paragraphe reprend des résultats exposés dans [13]. On suppose ici que $\mathcal{C}$ est une catégorie avec coproduits finis et produits fibrés. Les ensembles de morphismes de $\mathcal{Q C}$ sont munis d'une structure de monoïde pour la loi définie par

$$
(S \stackrel{f}{\longleftarrow} U \stackrel{g}{\rightarrow} T)+\left(S \stackrel{f^{\prime}}{\longleftarrow} V \stackrel{g^{\prime}}{\longrightarrow} T\right):=S \stackrel{f \sqcup f^{\prime}}{\longleftarrow} U \sqcup V \stackrel{g \sqcup g^{\prime}}{\longrightarrow} T
$$

Avec quelques hypothèses supplémentaires, la catégorie $\mathcal{Q C}$ est en fait semiadditive.

DÉFInItion 6.1. - Une catégorie avec coproduits et limites finies est un pseudo-topos si elle vérifie les conditions suivantes :

(i) tout morphisme vers un objet initial est un isomorphisme;

TOME $129-2001-\mathrm{N}^{\mathrm{O}} 2$ 
(ii) pour tous objets $A_{1}$ et $A_{2}$ et tout morphisme $X \rightarrow A_{1} \sqcup A_{2}$, si $X_{i}$, $i=1,2$, dénote le produit fibré

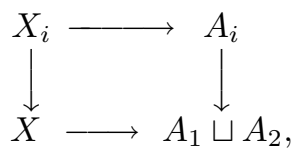

le morphisme $X_{1} \sqcup X_{2} \rightarrow X$ est un isomorphisme.

EXemple 6.2. — Tout topos élémentaire est un pseudo-topos. En particulier, la catégorie $\mathcal{E} n s$ est un pseudo-topos.

Proposition 6.3. - Soit $\mathcal{E}$ un pseudo-topos. La catégorie $\mathcal{Q} \mathcal{E}$ est semiadditive, et le foncteur $i_{*}: \mathcal{E} \rightarrow \mathcal{Q E}$ préserve le coproduit.

Démonstration. - Si $\phi$ est un objet initial de $\mathcal{E}$ et $\phi \stackrel{f}{\longleftarrow} V \stackrel{g}{\longrightarrow} T$ une flèche de $\mathcal{Q E}, f$ est un isomorphisme; ceci montre que $\phi$ est aussi un objet initial de $\mathcal{Q E}$. Soient maintenant $S_{1}$ et $S_{2}$ deux objets de $\mathcal{E}$ et $S_{1} \sqcup S_{2} \stackrel{f}{\longleftarrow} V \stackrel{g}{\rightarrow} T$ une flèche de $\mathcal{Q} \mathcal{E}$. Comme $\mathcal{E}$ est un pseudo-topos, $V=V_{1} \sqcup V_{2}$ et $f=f_{1} \sqcup f_{2}$, $f_{i}: V_{i} \rightarrow S_{i}, i=1,2$, et l'on a un isomorphisme

$$
\operatorname{Hom}_{\mathcal{Q E}}\left(S_{1} \sqcup S_{2}, T\right) \cong \operatorname{Hom}_{\mathcal{Q E}}\left(S_{1}, T\right) \times \operatorname{Hom}_{\mathcal{Q E}}\left(S_{2}, T\right) .
$$

Ceci montre que $S_{1} \sqcup S_{2}$ est le coproduit de $S_{1}$ et $S_{2}$ dans $\mathcal{Q} \mathcal{E}$, qui admet donc des coproduits finis. Par dualité, $\mathcal{Q} \mathcal{E}$ admet aussi des produits finis, l'objet $\phi$ est un objet nul de $\mathcal{Q} \mathcal{E}$ et le coproduit est isomorphe au produit. La catégorie $\mathcal{Q E}$ est donc semi-additive.

THÉORÈme 6.4. - La catégorie $\mathcal{Q} \mathcal{E}$ ns est équivalente à la catégorie $F(\mathbb{N})$ des monoïdes commutatifs libres de type fini.

Démonstration. - Comme tout ensemble fini est isomorphe à un coproduit de copies de $\underline{1}$, l'objet 1 est un générateur de $\mathcal{Q} \mathcal{E} n s$. Cette catégorie étant semi-additive, elle est équivalente à la catégorie $F\left(\operatorname{End}_{\mathcal{Q E} n s}(\underline{1})\right)$. Calculons le semi-anneau $\operatorname{End}_{\mathcal{Q E} n s}(\underline{1})$. Une classe d'équivalence de diagrammes $\underline{1} \leftarrow V \rightarrow \underline{1}$ est caractérisée par le cardinal de $V$, et $\operatorname{End}_{\mathcal{Q E} n s}(\underline{1})$ est donc le monoïde des entiers naturels. Comme le carré dans $\mathcal{E} n s$<smiles>[Tl][V][V][Tl]</smiles>

est cartésien, la composition correspond à la multiplication des entiers, ce qui achève la démonstration. 


\section{Démonstration du théorème 0.2}

On désire appliquer le théorème 2.1. Nous commençons par présenter une version pointée de la catégorie $\mathcal{Q} E n s$.

On notera $j$ le foncteur de $\mathcal{E} n s$ vers $\Gamma$ qui ajoute un point base $*$ à un ensemble fini. Si $X$ est un ensemble pointé, on note $X_{-}$le complémentaire du point base, et on convient que $j\left(X_{-}\right)=X$. Un morphisme $f: X \rightarrow Y$ est de la forme $j\left(f_{-}\right)$, pour une application $f_{-}: X_{-} \rightarrow Y_{-}$, si et seulement si $f^{-1}(\{*\})=\{*\}$. Plus généralement, tout morphisme $f: X \rightarrow Y$ se factorise de la manière suivante. Notons $X_{f}$ le complémentaire de $f^{-1}(*)$ dans $X$, et $f_{-}: X_{f} \rightarrow Y_{-}$l'application induite par $f$; l'inclusion $i_{f}: X_{f} \rightarrow X_{-}$induit $j\left(i_{f}\right): j\left(X_{f}\right) \rightarrow X$, qui a une rétraction $r_{f}: X \rightarrow j\left(X_{f}\right)$. On a $f=j\left(f_{-}\right) r_{f}$.

On définit une catégorie $\mathcal{Q}^{\prime}$ par générateurs et relations. Les objets de $\mathcal{Q}^{\prime}$ sont les ensembles finis pointés. Chaque application pointée $f: X \rightarrow Y$ fournit deux générateurs $f_{*}: X \rightarrow Y$ et $f^{*}: Y \rightarrow X$, et les relations sont les suivantes :

(i) $\quad(f g)_{*}=f_{*} g_{*}, \quad\left(1_{X}\right)_{*}=1_{X}, \quad(f g)^{*}=g^{*} f^{*}, \quad\left(1_{X}\right)^{*}=1_{X}$.

(ii) Si $i: X \rightarrow X \vee Y$ est l'inclusion et $r: X \vee Y \rightarrow X$ est la rétraction

$$
r_{*}=i^{*}, \quad r^{*}=i_{*} .
$$

(iii) Pour chaque carré cartésien de $\Gamma$

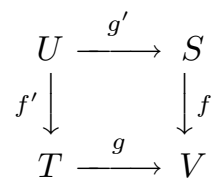

tel que $f^{-1}(\{*\})=\{*\}$,

$$
f^{*} g_{*}=g_{*}^{\prime} f^{\prime *} .
$$

On étend alors le foncteur $j$ en un foncteur :

$$
J: \mathcal{Q E} n s \longrightarrow \mathcal{Q}^{\prime}
$$

par $J(S)=j(S)=S \sqcup\{*\}$ et

$$
J(S \stackrel{f}{\longleftarrow} V \stackrel{g}{\longrightarrow} T)=j(g)_{*} j(f)^{*} .
$$

LEMme 7.1. - Le foncteur $J: \mathcal{Q E} n s \rightarrow \mathcal{Q}^{\prime}$ est une équivalence de catégories.

Démonstration. - Les remarques faites à propos du foncteur $j$ indiquent comment définir un quasi-inverse $J^{-1}$ au foncteur $J$. Sur les objets, $J^{-1}(X)=X_{-}$, et si $f: X \rightarrow Y$ est une application pointée, on pose

$$
J^{-1}\left(f_{*}\right):=X_{-} \stackrel{i_{f}}{\longleftarrow} X_{f} \stackrel{f_{-}}{\longrightarrow} Y_{-} \quad \text { et } \quad J^{-1}\left(f^{*}\right):=Y_{-} \stackrel{f_{-}}{\longleftarrow} X_{f} \stackrel{i_{f}}{\longrightarrow} X_{-} .
$$

Des vérifications sans surprise montre que ces formules définissent sur $\mathcal{Q}^{\prime}$ un foncteur $J^{-1}$, et qu'il s'agit d'un quasi-inverse de $J$.

TOME $129-2001-\mathrm{N}^{\mathrm{O}} 2$ 
La démonstration du théorème 0.2 se ramène ainsi à montrer que la catégorie $\mathcal{A} b^{\mathcal{Q}^{\prime}}$ est équivalente à la catégorie des foncteurs de Mackey de $\Omega$ vers $\mathcal{A} b$.

Soit donc $M$ un foncteur de $\mathcal{Q}^{\prime}$ vers $\mathcal{A} b$. Les relations (i) montrent que l'on obtient un foncteur de Janus de $\Gamma$ vers $\mathcal{A} b$ en posant $M_{*}(f):=M\left(f_{*}\right)$ et $M^{*}(f):=M\left(f^{*}\right)$. En appliquant le foncteur cr, on obtient un foncteur de Janus $\operatorname{cr}(M)$ de $\Omega$ vers $\mathcal{A} b$. Pour vérifier qu'il s'agit d'un foncteur de Mackey, considérons un carré de $\Omega$ cartésien dans $\mathcal{E} n s$ :

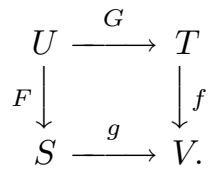

La relation (iii) de la définition de $\mathcal{Q}^{\prime}$ entraîne que

$$
M\left(j(f)^{*}\right) M\left(j(g)_{*}\right)=M\left(j(G)_{*}\right) M\left(j(F)^{*}\right) .
$$

Si l'on décompose $M(j(U))$ suivant ses déviations

$$
M(j(U))=\bigoplus_{X \subset U} \operatorname{cr}(M)(X)
$$

et que l'on suit $F$ et $G$ à travers cette décomposition, en utilisant les relations (ii), on obtient

$$
M\left(j(G)_{*}\right) M\left(j(F)^{*}\right)=\sum_{C(f, g)} \operatorname{cr}(M)_{*}\left(g^{\prime}\right) \operatorname{cr}(M)^{*}\left(f^{\prime}\right) .
$$

En appliquant le quasi inverse du théorème 2.1, on obtient la correspondance dans l'autre sens. Comme ces correspondances sont naturelles, il n'est plus très difficile de terminer la démonstration du théorème.

\section{Démonstration du théorème $\mathbf{0 . 3}$}

Soit $M$ un monoïde commutatif et $A$ un groupe abélien. Pour toute application $f$ de $M$ vers $A$ tel que $f(0)=0$, sa déviation, ou seconde déviation, est l'application de deux variables défini sur un couple $(x, y)$ de $M^{2}$ par

$$
f(x \mid y):=f(x+y)-f(x)-f(y) .
$$

Pour un entier $n \geq 2$, on définit par itération la $n$-ième déviation de $f$,

$$
\begin{aligned}
f\left(x_{1}|\cdots| x_{n-1} \mid x_{n}\right):=f\left(x_{1}|\cdots| x_{n-1}+x_{n}\right) & -f\left(x_{1}|\cdots| x_{n-1}\right) \\
& -f\left(x_{1}|\cdots| \hat{x}_{n-1} \mid x_{n}\right) .
\end{aligned}
$$

Une fonction polynomiale est une application dont l'une des déviations est nulle. On dit qu'une application est de degré $n$ si sa $(n+1)$-ième déviation est nulle, mais pas sa $n$-ième.

BULLETIN DE LA SOCiÉtÉ MATHÉMATIQUE DE FRANCE 
LEMME 8.1. - Soit $f: M \rightarrow A$ une fonction polynomiale d'un monoïde commutatif vers un groupe abélien. Il existe une unique extension $\operatorname{gr}(f)$ au complété en groupe $\operatorname{gr}(M)$ factorisant $f$ par l'adjoint de l'identité :

$$
\begin{gathered}
M \longrightarrow \operatorname{gr}(M) \\
f \searrow \swarrow \operatorname{gr}(f)
\end{gathered}
$$

L'application $\operatorname{gr}(f)$ est polynomiale de degré inférieur ou égal au degré de $f$.

On trouve une démonstration dans [8, Proposition 1.6]. la formule suivante convaincra le lecteur :

$$
\operatorname{gr}(f)(x-y)=\sum_{j \geq 1}(-1)^{j}(f(y|\cdots| y)-(x|y \cdots| y)),
$$

où le terme de rang $j$ dans la somme utilise la $j$-ième déviation de $f$. Cette somme a un sens dès que $f$ est polynomiale. En fait, l'application $\operatorname{gr}(f)$ a même degré que $f[11]$

Lemme 8.2. - Soient $n$ un entier positif et $\mathcal{A}$ une catégorie semi-additive. Un foncteur $T$ de $\mathcal{A}$ vers $\mathcal{A} b$ est polynomial de degré inférieur ou égal à $n$ si et seulement si pour toute paire d'objets $A, B$ de $\mathcal{A}$ l'application

$$
T: \operatorname{Hom}_{\mathcal{A}}(A, B) \longrightarrow \operatorname{Hom}(T A, T B)
$$

est polynomiale de degré inférieur ou égal à $n$.

Démonstration. — La démontration de [6, th. 9.11] s'applique.

Pour un semi-anneau $R$ et un entier positif $n$, on note $\mathcal{P}_{n}(R)$ la catégorie des foncteurs polynomiaux de $F(R)$ vers $\mathcal{A} b$ de degré inférieur ou égal à $n$. Les lemmes précédents entraînent :

Proposition 8.3. - Soient $R$ un semi-anneau et $n$ un entier positif. Les catégories $\mathcal{P}_{n}(R)$ et $\mathcal{P}_{n}(\operatorname{gr}(R))$ sont équivalentes.

Corollaire 8.4. - Tout foncteur polynomial de $F(\mathbb{N})$ vers $\mathcal{A} b$ a une extension unique à la catégorie $F(\mathbb{Z})$.

Preuve du théorème 0.3. - Le corollaire montre qu'il suffit de traiter le cas de foncteurs $M$ de $F(\mathbb{N})$ vers $\mathcal{A} b$ de degré $\leq n$. On remarque que dans la correspondance du théorème 0.2 , le foncteur $i_{*}$, respectant le coproduit (prop. 6.3), respecte le degré. Le foncteur $M_{*}$ de $\Gamma$ vers $\mathcal{A} b$ a donc même degré que $M$.

TOME $129-2001-\mathrm{N}^{\mathrm{O}} 2$ 


\section{Présentations des foncteurs de Mackey}

Dans ce paragraphe, nous désirons restreindre le nombre de relations de la définition des foncteurs de Mackey de $\Omega$ vers $\mathcal{A} b$ du paragraphe 5 .

Si $f: S \rightarrow T$ est une application entre ensembles finis, on appelle degré de $f$ en un point $t$ de $T$ le cardinal de la fibre au-dessus de $t$, et degré de $f$ le plus grand de ses degrés locaux :

$$
d(f):=\max \left\{\operatorname{Card} f^{-1}(\{t\}), t \in T\right\} .
$$

Le degré se comporte bien par produit fibré. En effet, pour un carré cartésien d'ensembles finis

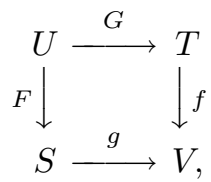

$U=S \times_{V} T$, on a, pour tout $s$ de $S$,

$$
F^{-1}(s)=\{s\} \times f^{-1}(g(s)) .
$$

Le degré de $F$ est donc inférieur ou égal à celui de $f$, et de même le degré de $G$ est inférieur ou égal à celui de $g$. A fortiori, pour un carré de la famille cartésienne de $(f, g)$,

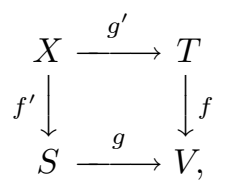

$X$ sous-ensemble de $U$, le degré de la surjection $f^{\prime}$ (respectivement $g^{\prime}$ ) est inférieur ou égal au degré de $f$ (resp. $g$ ).

Soit $M$ un foncteur de Janus de $\Omega$ vers $\mathcal{A} b$. Une paire de surjections de même but $f: T \rightarrow V$ et $g: S \rightarrow V$ est dite accessible à $M$ si, pour toute famille cartésienne $C(f, g)$,

$$
M^{*}(f) M_{*}(g)=\sum_{X \in C(f, g)} M_{*}\left(g^{\prime}\right) M^{*}\left(f^{\prime}\right)
$$

dans le groupe $\operatorname{Hom}(M(S), M(T))$. On dira qu'une surjection $f$ est accessible à $M$ (respectivement $n$-accessible à $M$ ) si pour toute surjection (resp. pour toute surjection de degré inférieur ou égal à $n) g$ les paires $(f, g)$ et $(g, f)$ sont accessibles à $M$. On dira enfin que $M$ est un foncteur de Mackey d'ordre $n$ si toute surjection est $n$-accessible à $M$.

Le cas des foncteur de Mackey d'ordre 1 est bien connu :

Lemme 9.1. - Soit $M$ un foncteur de Janus de $\Omega$ vers $\mathcal{A} b$. C'est un foncteur de Mackey d'ordre 1 si et seulement si pour toute bijection $f$, la composée $M_{*}(f) \circ M^{*}(f)$ est l'identité. 
Démonstration. - Si $f$ est une surjection de degré 1, c'est une bijection, et la famille cartésienne d'une paire contenant $f$ est réduite à un seul carré. En particulier, l'accessibilité de la paire $(f, f)$ se traduit sur le carré

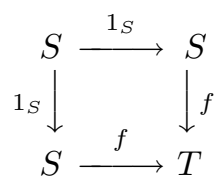

par la relation

$$
M^{*}(f) M_{*}(f)=1_{M(S)} .
$$

Réciproquement, si $f$ est une bijection et si

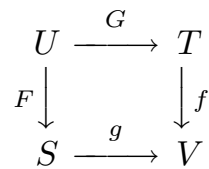

est un carré cartésien au-dessus d'une paire $(f, g)$, alors $F$ est aussi une bijection, et la commutativité du carré donne

$$
M_{*}(f) M_{*}(G)=M_{*}(g) M_{*}(F) .
$$

Si $M_{*}(f)=M^{*}(f)^{-1}$ et $M_{*}(F)=M^{*}(F)^{-1}$, on obtient :

$$
M_{*}(G) M^{*}(F)=M^{*}(f) M_{*}(g) \quad \text { et } \quad M_{*}(F) M^{*}(G)=M^{*}(g) M_{*}(f) .
$$

Les paires $(f, g)$ et $(g, f)$ sont donc accessibles à $M$.

Lemme 9.2. - Soit $M$ un foncteur de Janus de $\Omega$ vers $\mathcal{A} b$. La composée de deux surjections accessibles à $M$ (respectivement $n$-accessibles à $M$ ) est encore accessible à $M$ (resp. $n$-accessible à $M)$.

Démonstration. - Il s'agit d'une variation sur la composition des produits fibrés. Considérons un diagramme de carrés cartésiens

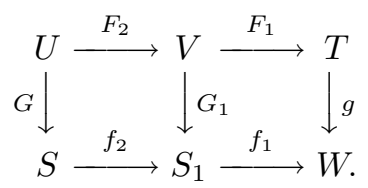

TOME $129-2001-\mathrm{N}^{\mathrm{O}} 2$ 
On a :

$$
\begin{aligned}
M^{*}(g) M_{*}\left(f_{1} \circ f_{2}\right) & =M^{*}(g) M_{*}\left(f_{1}\right) M_{*}\left(f_{2}\right) \\
& =\sum_{C\left(g, f_{1}\right)} M_{*}\left(f_{1}^{\prime}\right) M^{*}\left(g_{1}^{\prime}\right) M_{*}\left(f_{2}\right) \\
& =\sum_{C\left(g, f_{1}\right)}^{C} M_{*}\left(f_{1}^{\prime}\right) \sum_{C\left(g_{1}^{\prime}, f_{2}\right)} M_{*}\left(f_{2}^{\prime}\right) M^{*}\left(g^{\prime}\right) \\
& =\sum_{C\left(g, f_{1}\right)} \sum_{C\left(g_{1}^{\prime}, f_{2}\right)} M_{*}\left(f_{1}^{\prime} \circ f_{2}^{\prime}\right) M^{*}\left(g^{\prime}\right) \\
& =\sum_{C\left(g, f_{1} \circ f_{2}\right)} M_{*}\left(\left(f_{1} \circ f_{2}\right)^{\prime}\right) M^{*}\left(g^{\prime}\right),
\end{aligned}
$$

pourvu que la paire $\left(g, f_{1}\right)$ et les paires $\left(g_{1}^{\prime}, f_{2}\right)$ soient accessibles à $M$. La paire $\left(f_{1} \circ f_{2}, g\right)$ se traite par un calcul similaire.

Comme toute surjection est la composée de surjections de degré 2 , une conséquence immédiate du lemme 9.2 est :

LEMme 9.3. - Un foncteur de Mackey d'ordre 2 est un foncteur de Mackey.

Une surjection élémentaire est une surjection entre ensembles finis dont les cardinaux ne diffèrent que de 1.

LEMME 9.4. - Un foncteur de Mackey d'ordre 1 est un foncteur de Mackey d'ordre 2 si toute paire de surjections élémentaires de même but lui est accessible.

Démonstration. - Soit $(f, g)$ une paire de surjections, où $f$ est de degré 2 . On veut montrer qu'elle est accessible à $M$. Appliquant le lemme 9.2 à une décomposition de $g$ en produit de surjections élémentaires, on est ramené au cas où $g$ est une surjection élémentaire. Notons $x$ le point où $g$ est de degré 2 . Décomposons à son tour $f$ en produit de surjections élémentaires : $f=f_{n} \cdots f_{1}$, en supposant $f_{n} \cdots f_{2}$ de degré 1 en $x$. On procède alors par récurrence sur l'entier $n$. Pour le pas de récurrence, il suffit de remarquer que dans un carré cartésien :

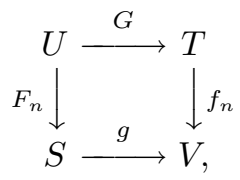

la surjection $G$ est élémentaire. On procède de la même manière pour la paire $(g, f)$.

Nous donnons maintenant des noms aux générateurs de notre présentation. Soit $n$ un entier positif. Pour chaque entier $i, 1 \leq i<n$, on note $s_{i}^{n}: \underline{n} \rightarrow \underline{n-1}$ 
l'unique surjection préservant l'ordre naturel telle que :

$$
s_{i}^{n}(i)=s_{i}^{n}(i+1) .
$$

Pour un foncteur de Janus $M: \Omega \rightarrow \mathcal{A} b$, notons $A_{n}$ le groupe abélien $M(\underline{n})$. On définit :

$$
\begin{aligned}
P_{i}^{n} & :=M_{*}\left(s_{i}^{n}\right): A_{n} \longrightarrow A_{n-1}, \\
H_{i}^{n} & :=M^{*}\left(s_{i}^{n}\right): A_{n-1} \longrightarrow A_{n} .
\end{aligned}
$$

Le groupe symétrique des bijections de $\underline{n}$ est désigné par $\mathfrak{S}_{n}$. La transposition de $\mathfrak{S}_{n}$ qui échange $i$ et $i+1$ est notée $\tau_{i}^{n}$. Commençons par rappeler une présentation de la catégorie $\Omega$.

LEMME 9.5. - La catégorie $\Omega$ est engendrée par les $\tau_{i}^{n}$ et $s_{i}^{n}, 1 \leq i<n$, soumis aux relations des groupes symétriques $\mathfrak{S}_{n}$ et aux relations supplémentaires suivantes :

$$
\begin{aligned}
s_{i} s_{j} & =s_{j-1} s_{i} & & \text { pour } i<j, \\
s_{i} \tau_{i} & =s_{i}, & & \\
\tau_{k} s_{j} & =s_{j} \tau_{k} & & \text { pour } k<j-1, \\
& =s_{j-1} \tau_{j} \tau_{j-1} & & \text { pour } k=j-1, \\
& =s_{j+1} \tau_{j} \tau_{j+1} & & \text { pour } k=j, \\
& =s_{j} \tau_{k+1} & & \text { pour } k>j .
\end{aligned}
$$

Proposition 9.6. - Soit $M$ un foncteur de Janus de $\Omega$ vers $\mathcal{A} b$. C'est un foncteur de Mackey si et seulement si les relations suivantes sont vérifiées pour tous les entiers $1 \leq i, j<n$ :

$$
\begin{aligned}
& T_{i}^{n}=M^{*}\left(\tau_{i}^{n}\right)=\left(M_{*}\left(\tau_{i}^{n}\right)\right)^{-1}, \\
& H_{j}^{n} P_{i}^{n}=P_{i}^{n+1} H_{j+1}^{n+1} \text { si } i<j, \\
& H_{j}^{n} P_{i}^{n}=P_{i+1}^{n+1} H_{j}^{n+1} \text { si } i>j, \\
& H_{i}^{n} P_{i}^{n}=1+T_{i}^{n}+P_{i}^{n+1} H_{i+1}^{n+1}+P_{i+1}^{n+1} H_{i}^{n+1} \\
&+P_{i+1}^{n+1} T_{i}^{n+1} H_{i+1}^{n+1}+P_{i}^{n+1} T_{i+1}^{n+1} H_{i}^{n+1} \\
& \quad+P_{i+1}^{n+1} P_{i}^{n+2} T_{i+1}^{n+2} H_{i}^{n+2} H_{i+1}^{n+1} .
\end{aligned}
$$

Démonstration. — Le lemme 9.1 dit que la relation (M1) traduit que $M$ est un foncteur de Mackey d'ordre 1. Le lemme 9.4 montre qu'il suffit d'écrire en outre la condition (M) pour les paires $\left(s_{i}^{n}, s_{j}^{n}\right)$ pour assurer que $M$ est un foncteur de Mackey d'ordre 2, et le lemme 9.3 montre que $M$ est alors un foncteur de Mackey. 
Dans le cas où $i<j$, la famille cartésienne de $\left(s_{i}^{n}, s_{j}^{n}\right)$ est réduite au seul carré

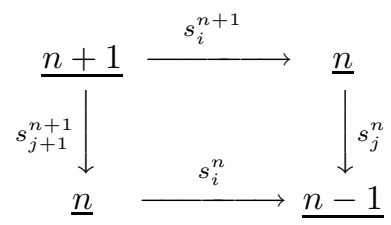

qui fournit la condition (M2). De même, le cas $i>j$ fournit la condition (M3). Dans le cas où $i=j$, outre le carré

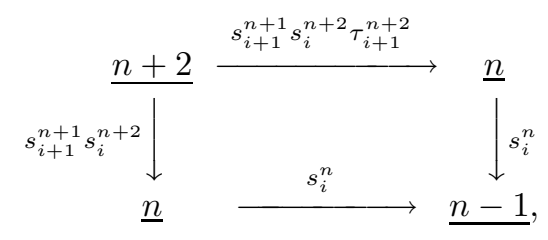

la famille cartésienne de $\left(s_{i}^{n}, s_{i}^{n}\right)$ contient les carrés correspondant aux sousensembles admissibles suivants de $\underline{n+2}$ :

$$
\begin{array}{lll}
\frac{n+2}{n}-\{i\}, & \underline{n+2}-\{i+1\}, & \underline{n+2}-\{i+2\}, \\
\underline{n+2}-\{i+3\}, & \underline{n+2}-\{i, i+3\}, & \underline{n+2}-\{i+1, i+2\} .
\end{array}
$$

On vérifie alors que la condition (M) équivaut à (M4).

Nous montrons maintenant comment se passer des groupes symétriques. Soit $\Delta^{*}$ la catégorie des ordinaux finis non vides et des surjections monotones. L'oubli de l'ordre est un foncteur de $\Delta^{*}$ vers $\Omega$. Le théorème 0.3 fournit donc un foncteur de la catégorie $\mathcal{P}_{n}(\mathbb{Z})$ vers la catégorie $\mathcal{J}_{n}\left(\Delta^{*}\right)$ des foncteurs de Janus de $\Delta^{*}$ vers $\mathcal{A} b$ qui s'annulent sur les ensembles ayant plus de $n$ éléments.

THÉORÈme 9.7. - Le foncteur $\mathcal{P}_{n}(\mathbf{Z}) \rightarrow \mathcal{J}_{n}\left(\Delta^{*}\right)$ induit par l'oubli de l'ordre est plein et fidèle.

Démonstration. - Soient $M$ et $M^{\prime}$ deux foncteurs de Mackey de $\Omega$ vers $\mathcal{A} b$ qui s'annulent sur les ensembles qui ont plus de $d$ éléments. Il suffit de montrer qu'une famille de morphismes de groupes $\alpha_{n}: A_{n} \rightarrow A_{n}^{\prime}, n \geq 1$, qui vérifient les conditions de naturalité

$$
\alpha_{n-1} P_{i}^{n}=P_{i}^{\prime n} \alpha_{n} \quad \text { et } \quad \alpha_{n} H_{i}^{n}=H_{i}^{\prime n} \alpha_{n-1}
$$

pour tous entiers $1 \leq i<n$, vérifie aussi les conditions

$$
\alpha_{n} T_{i}^{n}=T_{i}^{\prime n} \alpha_{n} .
$$

Comme $A_{n}=A_{n}^{\prime}=0$ quand $n>d$, ces conditions sont évidentes quand $n>d$. Comme la relation (M4) permet d'exprimer $T_{i}^{n}$ en fonction des applications 
$P_{i}^{k}, H_{i}^{k}$ et des applications $T_{i}^{k}$ pour $k>n$, on obtient la condition par une récurrence descendante sur l'entier $n$.

Remarque 9.8. - Ce dernier résultat montre qu'un foncteur polynomial de degré $\leq d$ est caractérisé par les groupes $A_{n}, n \leq d$, et les morphismes de groupes $P_{i}^{n}: A_{n} \rightarrow A_{n-1}, H_{i}^{n}: A_{n-1} \rightarrow A_{n}, 1 \leq i \leq n \leq d$. Les $T_{i}^{n}$ sont alors définis par récurrence à l'aide des relations (M4). Il n'est pas facile d'écrire les relations uniquement à l'aide des $P_{i}^{n}$ et des $H_{i}^{n}$ pour obtenir une présentation de $\mathcal{P}_{n}(\mathbb{Z})$ avec ces seuls générateurs-ci. Dans le cas $d=2$, on trouve [2] :

$$
H_{1}^{2} P_{1}^{2} H_{1}^{2}=2 H_{1}^{2}, \quad P_{1}^{2} H_{1}^{2} P_{1}^{2}=2 P_{1}^{2},
$$

et pour $d=3[4]$ :

$$
\begin{aligned}
& H_{1}^{3} H_{1}^{2}=H_{2}^{3} H_{1}^{2}, \quad P_{1}^{2} P_{1}^{3}=P_{1}^{2} P_{2}^{3}, \quad H_{2}^{3} P_{1}^{3}=0, \quad H_{1}^{3} P_{2}^{3}=0, \\
& H_{1}^{3} P_{1}^{3} H_{1}^{3}=2 H_{1}^{3}, \quad P_{1}^{3} H_{1}^{3} P_{1}^{3}=2 P_{1}^{3}, \\
& H_{2}^{3} P_{2}^{3} H_{2}^{3}=2 H_{2}^{3}, \quad P_{2}^{3} H_{2}^{3} P_{2}^{3}=2 P_{2}^{3}, \\
& H_{1}^{2} P_{1}^{2} H_{1}^{2}=2 H_{1}^{2}+2\left(P_{1}^{3}+P_{2}^{3}\right) H_{1}^{3} H_{1}^{2}, \\
& P_{1}^{2} H_{1}^{2} P_{1}^{2}=2 P_{1}^{2}+2 P_{1}^{2} P_{1}^{3}\left(H_{1}^{3}+H_{2}^{3}\right), \\
& H_{1}^{3} H_{1}^{2} P_{1}^{2}+H_{1}^{3}+H_{2}^{3}=H_{2}^{3} P_{2}^{3} H_{1}^{3} P_{1}^{3} H_{2}^{3}+H_{1}^{3} P_{1}^{3} H_{2}^{3} P_{2}^{3} H_{1}^{3}, \\
& H_{1}^{2} P_{1}^{2} P_{1}^{3}+P_{1}^{3}+P_{2}^{3}=P_{2}^{3} H_{1}^{3} P_{1}^{3} H_{2}^{3} P_{2}^{3}+P_{1}^{3} H_{2}^{3} P_{2}^{3} H_{1}^{3} P_{1}^{3} .
\end{aligned}
$$

\section{BIBLIOGRAPHIE}

[1] Bass (H.) - Algebraic K-theory, Benjamin, New York, 1968.

[2] Baues (H.J.) - Quadratic functors and metastable homotopy, J. Pure Appl. Algebra, t. 91 (1994), p. 49-107.

[3] BÉnabou (J.) - Introduction to bicategories, in Reports of the Midwest Category Seminar, Springer Lecture Notes in Math., 47 (1967), p. 1-77.

[4] Dreckmann (W.) - A sketch for polynomial functors, in Actes des journées mathématiques Catégories, algèbres, esquisses et néoesquisses, Caen 1994, p. 91-95.

[5] Dress (A.) - Contributions to the theory of induced representations, in Algebr. K-Theory II, Proc. Conf. Battelle Inst. 1972, Springer Lecture Notes in Math., 342 (1973), p. 183-240.

[6] Eilenberg (S.), Mac Lane (S.) - On the groups $H(\pi, n)$ II, Ann. of Math., t. 60 (1954), p. 49-139.

[7] Franjou (V.), Pirashvili (T.) - en préparation.

[8] Joukhovitski (S.) - K-theory of the Weil transfer functor, K-Theory, t. 20 (2000), p. $1-21$ 
[9] Jibladze (M.), Pirashvili (T.) - Cohomology of algebraic theories, J. Algebra, t. 137 (1991), p. 253-296.

[10] Linder (H.) - A remark on Mackey-functors, Manuscripta Math., t. 18, $\mathrm{n}^{\mathrm{o}} 3$ (1976), p. 273-278.

[11] Müller (H.) - Algebraische Abbildungen, Dissertation. Universität Bielefeld (1971)

[12] Pirashvili (T.) - Dold-Kan type theorem for $\Gamma$-groups, Preprint Universität Bielefeld, 1998.

[13] Thévenaz (J.), WeBB (P.) - The structure of Mackey functors, Trans. Amer. Math. Soc., t. 347, no 6 (1995), p. 1865-1961. 